Military Technical College

Kobry El-Kobbah,

Cairo, Egypt

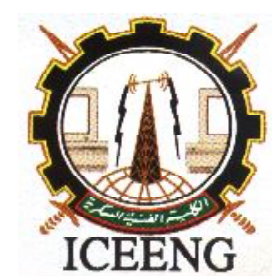

$9^{\text {th }}$ International Conference

on Electrical Engineering

ICEENG 2014

\title{
Exploration of the Brain and Cardiovascular System Interactions under Physical Stress
}

By

Ehab Shaaban $^{1} \quad$, Emad Elsamahy $^{1}$, Mahmoud Gaddallah $^{1} \quad$ and Ahmed Genedy ${ }^{2}$

\section{$\underline{\text { Abstract: }}$}

The brain activity is a very complex process, which is mainly characterized by the electroencephalogram (EEG). Moreover, there exist many interactions between the brain and other human body systems such as the cardiovascular system. Therefore, to explore these interactions a model was identified based on intelligent modeling tools (fuzzy-ARX). Signals generated from a previous work related to the modeling of the cardiovascular system were used as an input for the proposed model. EEG signals were measured for six male subjects and were used as an output for the proposed model. The model output was validated in both time and frequency domains for each subject. Furthermore, the validation results revealed that there exist good match between the measured EEG signals and the corresponding estimated EEG signals generated from the proposed model for each subject. Moreover, the model could be used as a descriptor for the relationships existed between the brain and the cardiovascular system afferents.

\section{Keywords:}

Intelligent modeling tools, Cardiovascular System, Electroencephalogram (EEG) , Brain Activity.

1 Biomedical Engineering Department, Military Technical College, Cairo, Egypt

2 Medical Services Administration, Cairo, Egypt 


\section{Introduction:}

The human operator's role has become more difficult with increasing operational demands, stress and fatigue, with a consequent threat to safety and reliability [1]. In this research work, a proposed model was proposed to predict the interactions between the brain and the cardiovascular system (CV). This model will be able to predict the electroencephalogram (EEG) signal based on the afferent signals generated from the cardiovascular system model in a previous work. These signals are estimated for controlling the correct function of the cardiovascular system. Furthermore, they are generated from the various sensors distributed throughout the human body for detecting the variation of heart rate (HR), blood pressure (BP) and respiration (RESP) signals under various circumstances [2].

Heart rate is controlled by the autonomic nervous system through two branches of effectors: sympathetic and parasympathetic (vagus) fibers. These two fibers are controlled through the cardiac center in the brain. Additionally the vasodilatation and vasoconstriction of blood vessels change the blood pressure, which are controlled by the vasomotor center in the brain [2]. Moreover, it has been found that the respiration (RESP) signal, which is controlled by the respiratory center, introduces some disturbances to the blood pressure [2].

Luczak and Raschke [4] proposed a closed-loop model to represent the cardiovascular system and to explore the interactions between the cardiovascular and respiration systems. In 1980, Luczak et al. developed their second CV model with some modifications mainly in the respiratory module [5]. Other researches were carried out a study to explore the effect of physical stress on subjects in order to construct a detailed closed loop CV model [3].

Since, the EEG signal is a very complicated signal, a simpler version, which is the evoked potential, is used in order to study its relation to other signals.

The evoked potential signal is the average behavior over multiple EEG cycles in response to repetitive stimuli (visual or auditory). Several researchers used this signal in their work such as Robinson [6], and Jansen and Rit [7]. Consequently, Robinson [8, 9] established the existence of a correlation between the evoked potential signal and the responses of the brainstem and hypothalamus $[8,9]$.

The paper is organized as follows: Section 2 discusses the modeling identification tools; Section 3 states the proposed model implementation and validation results. Finally, Section 4 states the conclusion and future research work. 


\section{Modeling Identification Tools:}

Various modeling identification tools can be used in identifying the proposed model such as Neuro-fuzzy tool, and Fuzzy-ARX tool. These tools were selected because of their accurate results in identifying models. Before outlining the obtained results, a brief discussion will be given of all these methods. The neuro-fuzzy tool has been widely and successfully used during the last decade in many applications such as system identification and control. It is used as a non-linear method for mapping a certain number of inputs to a certain number of outputs. The two most popular types of fuzzy rules processing are the Mamdani type and the Sugeno-type. The general form of the two types of rules is given below:

Mamdani:

$\mathrm{R}^{\mathrm{i}}$ : IF $x_{1}$ is $A_{i 1}$ and $x_{2}$ is $A_{i 2}$ and $\ldots$ and $x_{m}$ is $A_{i m}$ THEN $y_{i}$ is $B_{i}$

Sugeno:

$\mathrm{R}^{(\mathrm{i})}: \operatorname{IF} x_{1}$ is $A_{i 1}$ and $x_{2}$ is $A_{i 2}$ and $\ldots$ and $x_{m}$ is $A_{i m}$ THEN $y_{i}=\mathrm{f}\left(x_{1}, x_{2}, . ., x_{m}\right)$

Where:

- $\left(x_{1}, x_{2}, \ldots, x_{m}\right)$ are the inputs to the system.

- $y$ is the output.

- $A_{i l}, A_{i 2}, A_{i m}$, and $B_{i}$ are linguistic labels such as: zero (ZE), negative small (NS), positive big (PB) etc.

- $f\left(x_{1}, x_{2}, \ldots, x_{m}\right)=c_{0}+c_{1} x_{1}+c_{2} x_{2}+\ldots+c_{n} x_{n}$, i.e. A linear function.

- $R^{(i)}$ denotes that the above is the $i^{\text {th }}$ rule in the rule-base.

- $i=1,2, \ldots, M$, where $M$ is the number of rules.

It can be seen that in the Mamdani type of fuzzy rules processing, both the antecedent (IF) and the consequent (THEN) parts are fuzzy, while in sugenotype the consequent part is not fuzzy but a deterministic (static or dynamic) function, which can either be linear or non-linear.

The Fuzzy-ARX tool, as implied by its name, combines both the fuzzy interpretation of the inputs through a number of membership functions and the ARX model. The advantage of such a structure is its simple implementation as well as its uniqueness such that it needs no further tuning, in terms of modifying the parameters of the membership functions in the input space, to achieve better results. This structure is similar to the Sugeno-type, which was previously discussed, in that its consequent-part is an ARX model rather than a membership function as in the Mamdani type. This tool, with a second-order ARX model, has the following rules-representation: 
$\mathrm{R}^{(\mathrm{i})}: \operatorname{IF} u$ is $A_{i}$ THEN $\left.y(\mathrm{t}+1)=-a_{1}{ }^{(i)} y(t)-a_{2}^{(i)} y(t-1)\right)+b_{1}^{(i)} u(t)+b_{2}^{(i)} u(t-1)+$ offset

Where:

- $u$ is the input to the system.

- $y$ is the output of the system.

- Ai is a linguistic label such as: zero (ZE), negative small (NS),

- $\mathrm{Ai}$ is a linguistic label such as: zero (ZE), negative small (NS), positive big (PB), etc. $\mathrm{R}(\mathrm{i})$

- $R^{(i)}$ denotes that the above is the $i^{\text {th }}$ rule in the rule-base.

- $i=1,2, \ldots, M$, where $M$ is the number of rules.

Offset represents the fact that some signals start from a significant baseline value.

A detailed comparison was carried out between the estimated EEG and the actual EEG signals with various parameters using the two mentioned modeling tools. The results revealed that the best modeling tool to be used for identifying the proposed model is the Fuzzy-ARX tool in terms of the prediction accuracy.

\section{Model Implementation:}

It is well known that the EEG signal, which can be measured on the surface of the scalp, includes the various components relating to all parts of the brain. These components are control signals to certain parts of the body as well as signals received from other parts of the body as a feedback sensory. Thus, according to this fact these signals should be acquired in a certain way that ensures appropriateness of signals, which facilitate the process of identifying a model representing the process of generating the EEG signal. In other words, the signals which are transmitted and received through the nerve fibers, emitted from the brain, must be acquired. However, since it was very difficult to acquire such signals from subjects undergoing the experiments. Therefore, in order to simplify the identification process, the nerve impulses that where generated from a previous model that was identified based on Luczak first and second models [3] where used as a representation for these signals (inputs).Moreover, the EEG signals for subjects under physical stress were used as output.

The physical stress was designed as a sinusoidal stimulus applied to a pc-controlled bike. The frequency of this stimulus was selected to be $0.0909 \mathrm{~Hz}$ to test for the occurrence of BP entrainment (changing the spontaneous BP oscillation from $0.1 \mathrm{~Hz}$ to the stimulus frequency which ensures the full transmission of the stimulus to the subject under test. 


\section{$\underline{3.1 \text { Preprocessing of input and output signals }}$}

The proposed model will be implemented based on a phenomenon called "phase locking" which was discovered between the EEG signal on one hand and the BP,RESP signals on the other hand within their frequencies ranges ( BP: 0.08-0.12) and ( RESP:0.4-0.6 ).

Therefore, some afferent signals were selected due to their initiate relation to BP and RESP signals as inputs to the proposed model, which are sympathetic, parasympathetic, respiration and vasomotor impulses. Some preprocessing was applied to the input and output signals in order to be used for the proposed model identification. The preprocessing phase was divided into two parts, which are:

\subsubsection{Signal conditioning of the EEG signal:}

The used EEG signal was acquired from the $C_{z}$ according to the 10-20 system. The signals measured from this location was found to be highly correlated to the activities of the thalamus and brain stem [8,9].Therefore, Sympathetic, Parasympathetic, Respiration and vasomotor was selected as there centers are originated in the brain stem. Since, the cardiovascular signals frequency ranges are always below $1 \mathrm{~Hz}$ (BP: $0.1 \mathrm{~Hz}$ and RESP: 0.4-0.6 Hz). Therefore, wavelet multi-level decomposition was applied to the EEG signal to extract the delta band $(0-4 \mathrm{~Hz})$ using "Daubechies" filter with the order 24. Moreover, a down sampling phase to $2 \mathrm{~Hz}$ was applied to the EEG signal as shown in Figure (1).

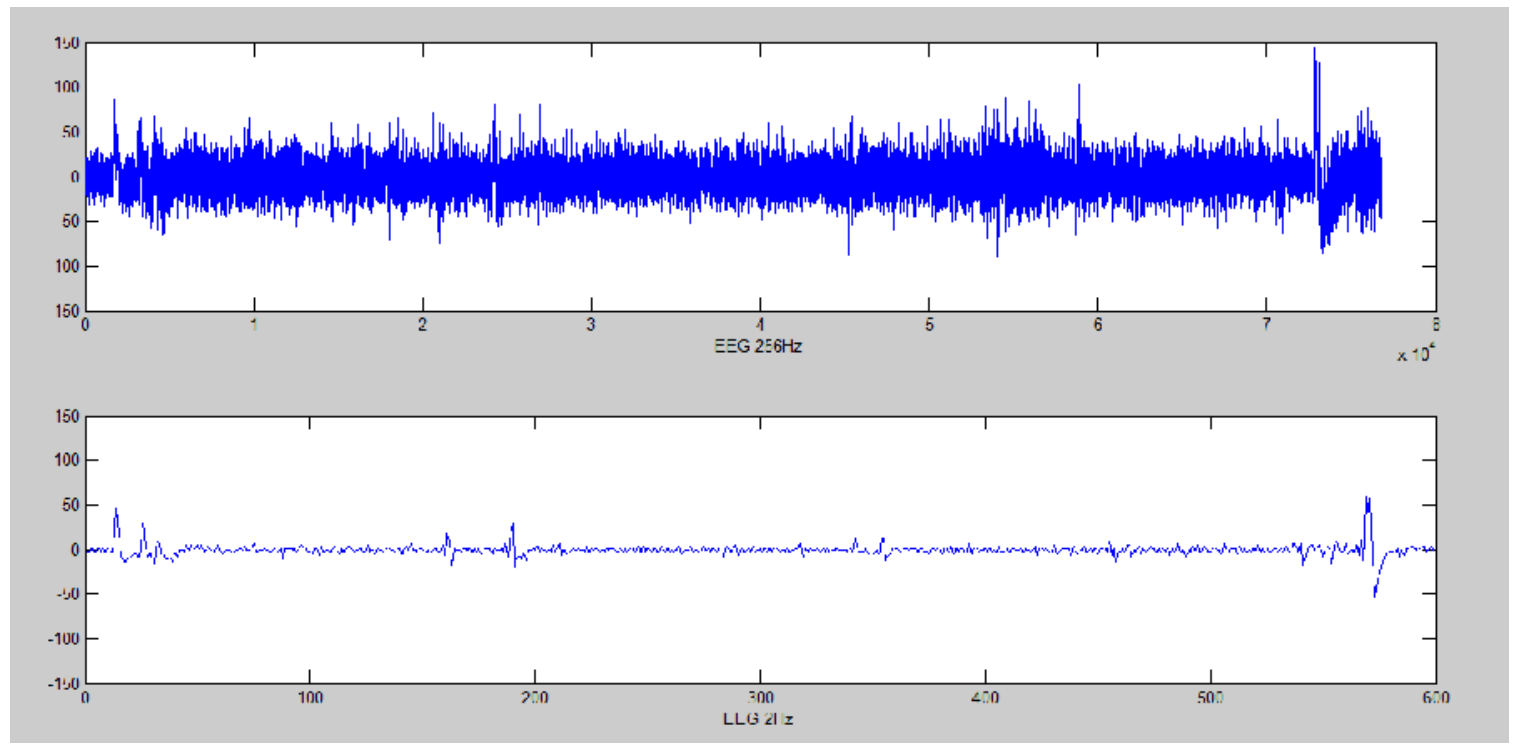

Figure (1): The actual EEG vs delta band at $2 \mathrm{~Hz}$ 
As a final step of the preprocessing two ranges where selected from the EEG delta band which corresponds to the useful range for BP and RESP signals (BP: 0.08-0.12 Hz and RESP: 0.4-0.6 HZ ) the rest of the signal was considered as background noise to be added later after EEG estimation as shown in Figure (2).

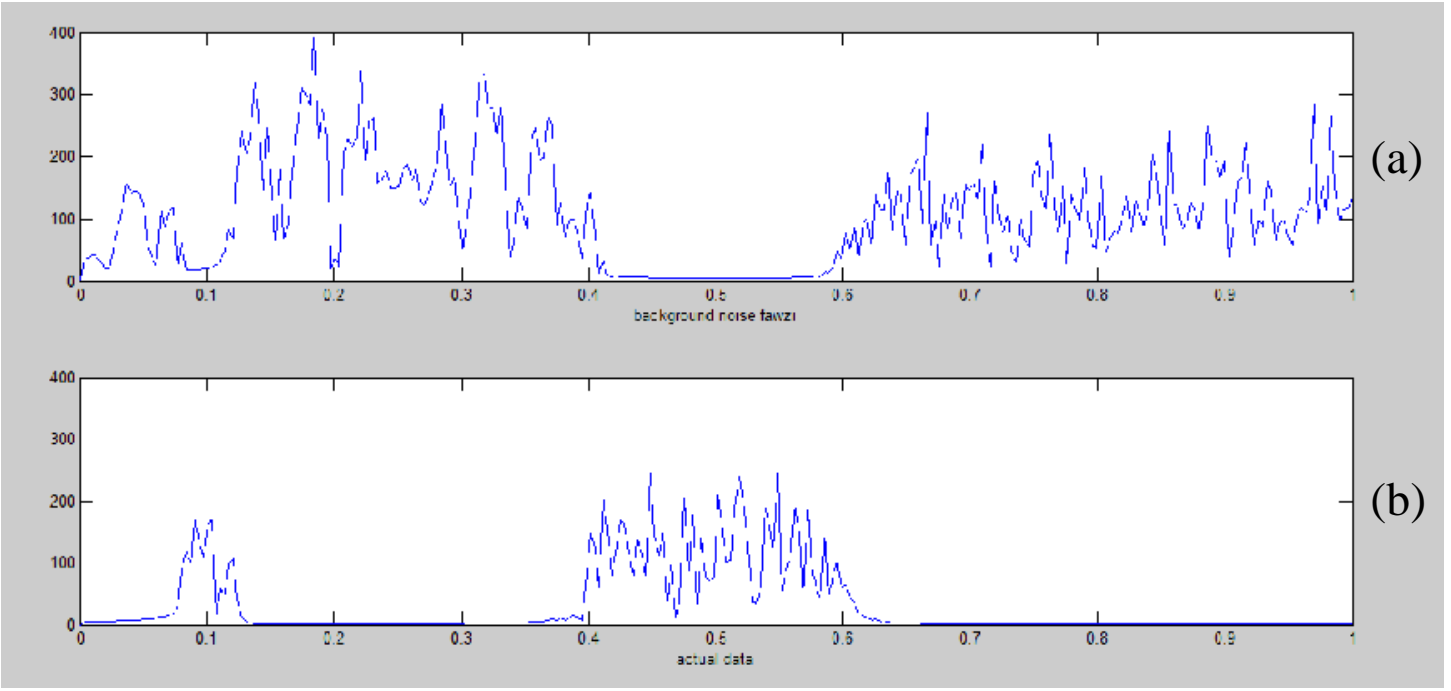

Figure (2): (a) background noise, (b) EEG in useful ranges

\subsubsection{Signal Conditioning of the input afferent impulses:}

The input afferent impulses used in this proposed model where extracted from a previously implemented cardiovascular model for subjects under physical stress [3].Some pre-processing techniques where applied to these signals such as:

- Re-sampling to $2 \mathrm{~Hz}$.

- Removing the signal trend, which corresponds to a high pass filter to remove the unwanted low frequency components.

Moreover, Figure (3) shows the used afferent signals as inputs for the proposed EEG model. 


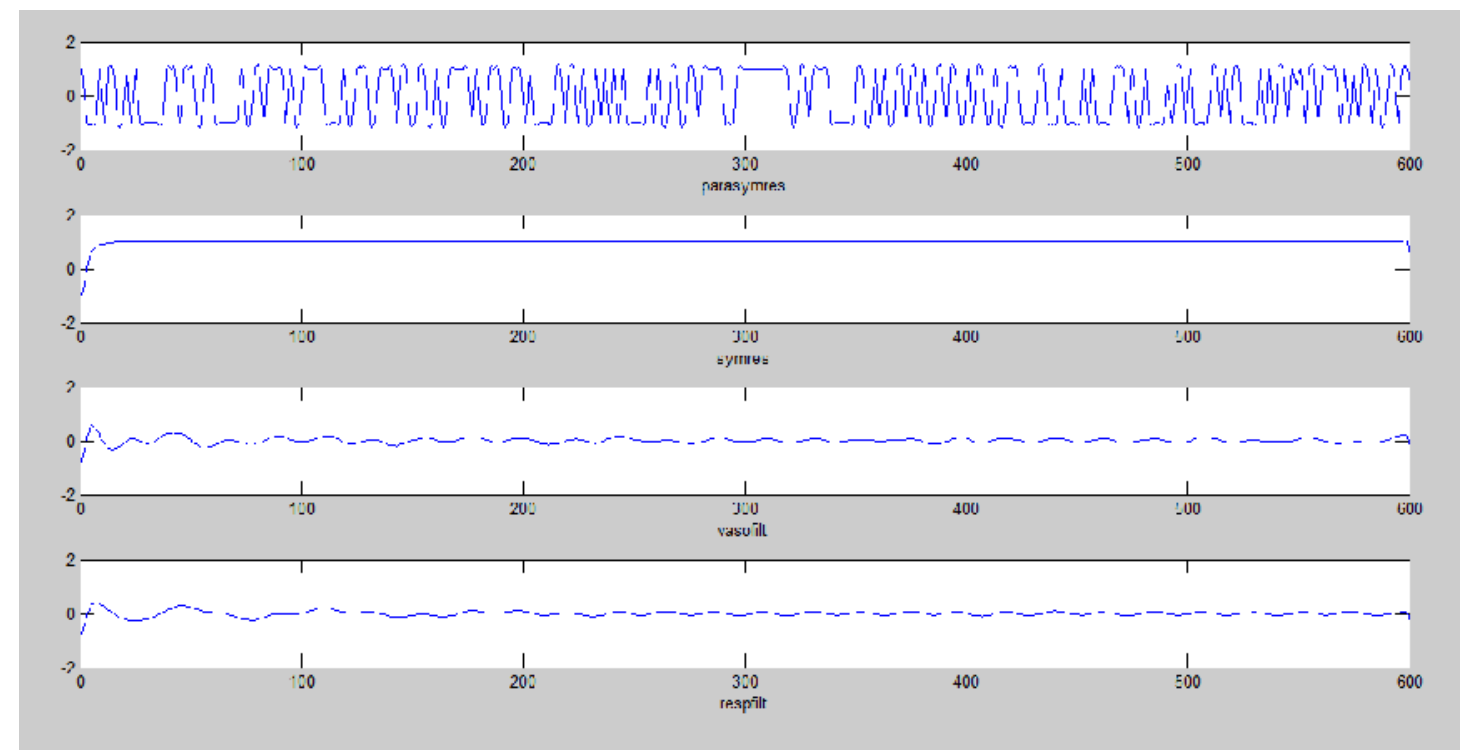

Figure (3): Input afferent signals.

\subsubsection{Model Construction:}

The inputs shown in Figure (3) and the output shown in Figure 4 where used as inputs and output for the EEG model construction using the Fuzzy-ARX tool which was selected according to the results previously mentioned. Various parameters for this tool where tested in order to achieve the best accuracy. The constructed model was tested against the actual data as shown in the following section.

\subsubsection{Model Validation}

The estimated EEG signal was tested in the time domain using (MSE) as well as in the frequency domain as shown in Figure (4) and Figure (5) respectively. 


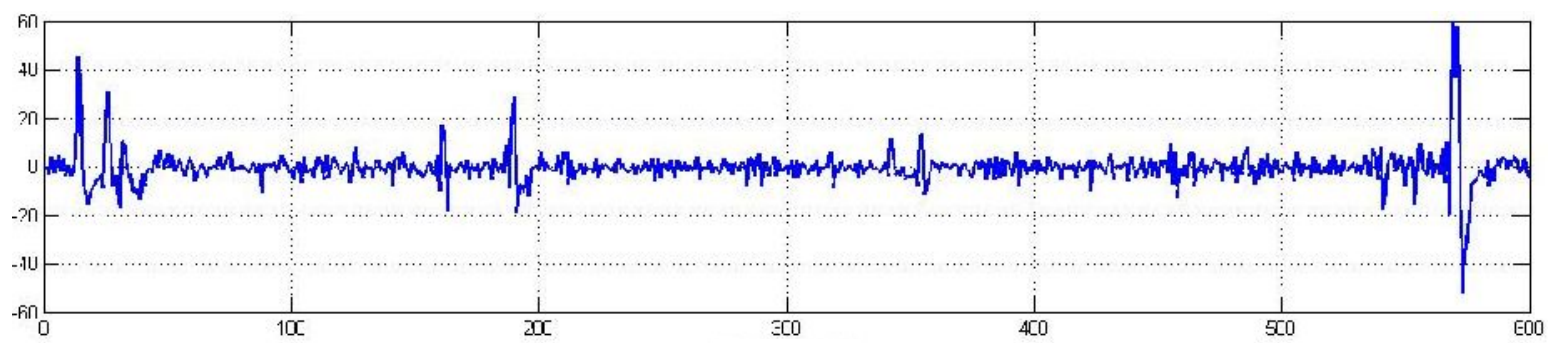

(a)

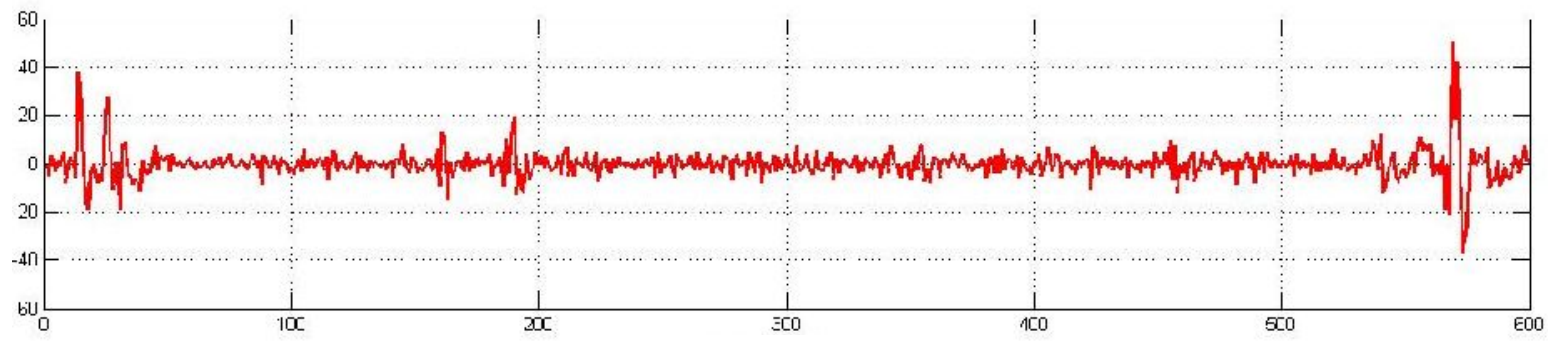

(b)

Figure (4): Actual (a) vs. estimated (b) EEG signals in time domain.

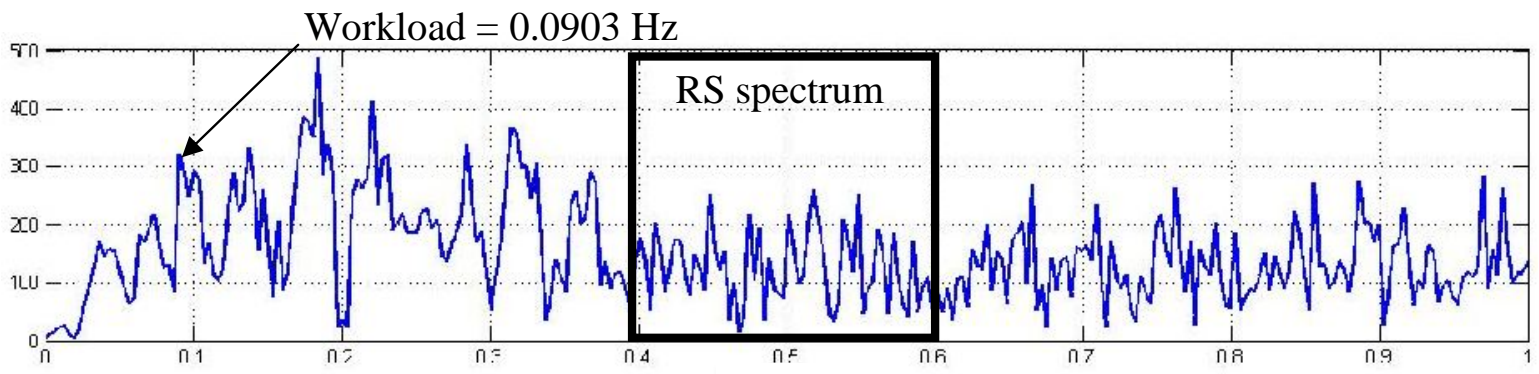

(a)

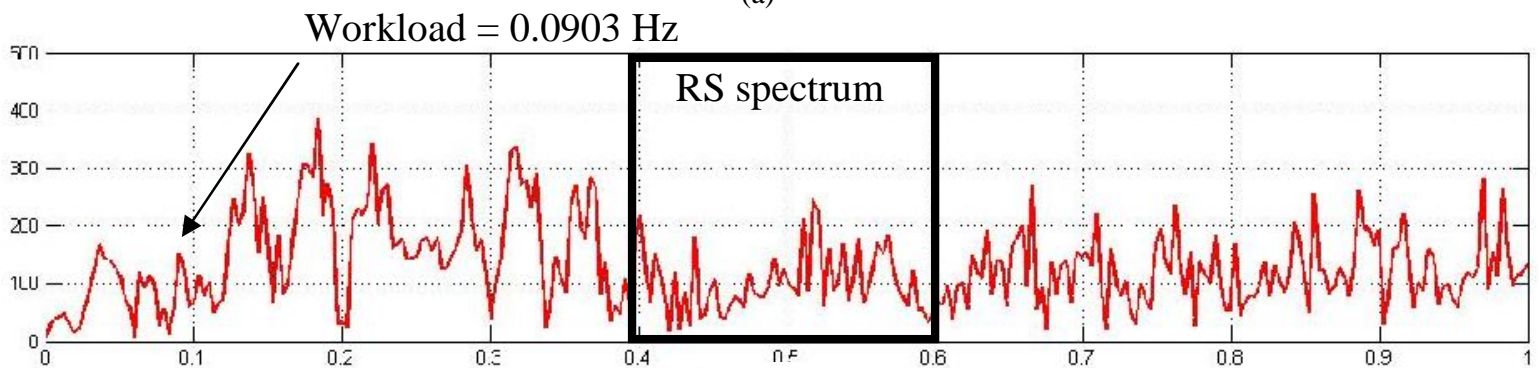

(b)

Figure (5): Actual (a) vs. estimated (b) EEG signals in frequency domain.

Furthermore, the results showed that there exist good match between the actual and estimated EEG signals as can be seen in the previous Figures. 


\section{Conclusions:}

The proposed model succeeded in describing the interactions between the brain and cardiovascular system and other control systems such as respiratory system. The existence of the brain centers highlighted the essential role of these centers in preserving the correct operation for these control systems under various perturbations such as physical stress. The fuzzy-ARX modelling method was chosen in our work because it provides high accuracy in the case of signals containing high frequencies, such as the EEG signal. The estimated output of the model has been validated against the actual data in both time and frequency domains and it shows good accuracy in estimating these signals in both domains. The obtained results could be used as a basis for the construction of generic EEG model. Moreover, the EEG model parameters could be used in the field of telemedicine for generating the EEG signals of subjects who lived in rural areas to be investigated in far large hospitals without the need of subject attendance.

\section{References:}

[1] Hancock PA, Desmond PA, editors. "Stress, workload and fatigue”. Mahwah, NJ: Erlbaum; 2001.

[2] Thibodaux GA, Patton KT. "Physiology of the cardiovascular system” London: Mosby Books; 1999.

[3] Mohamed A. Abbass "Modeling of electrical activities in cardio-respiratory system " Msc. in electrical engineering,cairo,2012.

[4] Luczak H, Raschke F. "A model of the structure and the behaviour of human heart rate control”. Biol Cybernet1975; 18:1-13.

[5] H. Luczak, U.Philipp and W.Rohmert, "Decomposition of Heart-Rate Variability under the Ergonomic aspect of Stressor analysis," in The Study of heart-rate variability, R. I. KITNEY, O.ROMPELMAN, Ed., ed New York: Oxford University, 1980.

[6] Robinson DL. "An anlysis of human EEG responses in the alpha range of frequencies". Int J Neurosci 1983;22:81-98.

[7] Jansen BH, Rit VG." Electroencephalogram and visual evoked potential generation in a mathematical model of coupled cortical columns". Biol Cybernet 1995; 73: 357-66.

[8] Robinson DL. 'The Technical, neurological, and psychological significance of 'alpha', 'delta', and 'theta' waves confounded in EEG evoked potentials": a study of peak latencies. Clinical Neurophysiology 1999; 110:1427-34.

[9] Robinson DL. 'The Technical, neurological, and psychological significance of 'alpha', 'delta', and 'theta' waves confounded in EEG evoked potentials: a study of peak amplitudes", Personal Individual Differ 2000; 28:673-93.

[10] E. Elsamahy, "A Generic Grey-box Model for the Cardiovascular System of Subjects Experiencing Physical Stress," Doctor of Philosophy, Automatic Control and Systems Engineering, Sheffield, 2003. 